\title{
Measurement and characterization of dual-band LoRa communication in the Antarctic
}

\author{
Patrick Van Torre ${ }^{1}$, Johnny Gaelens ${ }^{1}$, Jo Verhaevert ${ }^{1}$, Hendrik Rogier ${ }^{1}$ \\ ${ }^{1}$ Ghent University - imec, IDLab, Department of Information Technology (INTEC), \\ Technologiepark-Zwijnaarde 15, 9052 Gent, Belgium, patrick.vantorre@ugent.be
}

\begin{abstract}
The Antarctic is vast continent without any communication or power infrastructure. However, many countries operate scientific experiments in the Antarctic and desire lowpower long-range data communication for their scientific measurement setups. Currently, expensive satellite communication via Argos or Inmarsat is often employed. In case of limited data rate, sub-GHz frequency bands offer excellent radio wave propagation to provide ground-based communication links. The longer wavelength in these bands results in less path loss for a similar range, compared to microwave links at $\mathbf{G H z}$ frequencies. In a recent evolution, LoRa networks are deployed, allowing a larger link budget thanks to chirp spread spectrum modulation. In this paper, a number of dual band mobile-to-base-station measurements are described for the 434 and $868 \mathrm{MHz}$ frequency bands. The channel measurements are analyzed for signal to noise ratio and packet loss, as recorded for different practical trajectories. The relation between packet loss and signal to noise ratio is also provided for practical applications in mobile to base station communication links.
\end{abstract}

Index Terms-Antarctic, LoRa, antenna, propagation, measurement.

\section{INTRODUCTION}

The Antarctic is still a mostly unexplored environment situated far away from the inhabited world. Scientific base stations [1], experimental sensor setups and mobile vehicles employed for expeditions all need wireless data communication. In this continent at this moment no frequency regulation exists for radio communication, implying that theoretically all frequency bands could be used without power limit. Of course, it is important to employ as little power as possible in order to extend the battery life of remote equipment. Renewable energy is generally preferred, charging the batteries of long-term remote science setups by means of solar power or wind energy. Additionally, despite the freedom offered by the unregulated spectrum, it is technically more feasibly to employ technology which is already validated in the inhabited world.

The range for which communications need to be established is in the order of up to almost $30 \mathrm{~km}$ around the base station. In the field of wireless sensor networks, an evolution exists towards the use of sub-GHz frequencies for long-range low-power communication. Sub-GHz frequencies offer the advantage of a longer range, combined with more diffraction of the waves around small obstacles. Different technologies are currently being promoted and used in industry, of which the most popular are LoRa [2], Sigfox [3] and DASH7 [4]. In a recent publication LoRa technology was employed in the
Antarctic, resulting in successful data communication up to a distance of $28 \mathrm{~km}$ between the base station and a mobile vehicle [5].

LoRa technology is indeed a promising technique for longrange links, allowing low packet loss, even with very weak signals buried in the noise, thanks to the chirp spread spectrum modulation (CSS) providing a large spreading gain. An alternative for long-range links is Sigfox, but in comparison LoRa offers a larger bandwidth and data rate. The adaptive data rate is considered an advantage, increasing the autonomy of the wireless nodes. Transmissions can indeed be shorter to transmit a given amount of data, resulting in optimal energy consumption. Additionally, the larger bandwidth and CSS modulation employed by LoRa allows the system to handle Doppler shifts of the signals more efficiently, resulting in better mobile performance. The DASH7 standard, in comparison, offers a data rate comparable to LoRa but is presented as a solution for link ranges up to $2 \mathrm{~km} \mathrm{[4].}$

The Antarctic, with it's thick ice sheet, mountains and absence of buildings, presents an interesting environment for propagation measurements. Channel characterization, as often performed in the inhabited world [6], is not found in literature for this continent.

Recent publications covering LoRa experiments are mostly performed in cities, where interference is an important performance-limiting issue [7]. Indoor as well as outdoor measurements with LoRa links are described in [8], providing interesting information about propagation over water for a range comparable to measurements following in this paper.

A first set of Antarctic channel measurements was presented in [5]. In this paper measurements for wireless mobile to base-station links are documented and compared for different frequency bands. This paper focuses on the relation between Signal-to-Noise Ratio (SNR) and packet loss for different practical mobile-to-base-stations links. Measurements are repeated and compared for similar trajectories. The characteristics for measurements performed on different days are compared. Additionally, measurements in different bands are analyzed in order to obtain a general performance indicator, comparing the efficiency of those bands as a candidate for wireless sensor communication in the Antarctic. The packet loss is also determined as a function of SNR for practical links.

The paper is further organized as follows. The measurement setup is presented in Section II, the measurement results in Section III and finally by the conclusions in Section IV. 


\section{Measurement Setup}

\section{A. General setup}

The general setup is valid for both the base station and the mobile vehicle. Measurements are performed employing LoRa communication from a mobile transmitter to a receiving base station in both the 434 and $868 \mathrm{MHz}$ bands.

Packets are sent every 5 seconds, alternating on each band in order to allow a performance comparison in both bands for the distance covered by the mobile vehicle. The communication is one-way, without acknowledge packets sent by the receiver. GPS positions are continuously recorded, providing accurate range information.

The Microchip Technology DM164138 board [9] is employed as a dual-band transceiver at both sides of the link. This board relies on the RN2483 LoRa chip. LoRa modulated packets with a 3-byte payload containing increasing sequence numbers are transmitted at a power of $+14 \mathrm{dBm}$, with $125 \mathrm{kHz}$ bandwidth, 4/5 coding rate, a preamble length of 10 chips and a bandwidth spreading factor of 12 . A Cyclic Redundancy Check (CRC) is also employed, guaranteeing the correctness of the received packet's data content. These parameters correspond to the board's default configuration, resulting in a data rate of $293 \mathrm{bps}$.

\section{B. Base station receiving setup}

At the Belgian Princess Elisabeth Antarctic Station [1], a dual band LoRa receiver is installed, connected to vertically polarized directional Yagi-Uda antennas installed on the roof of the station, as illustrated in Fig. 1. The detailed picture in Fig. 2 shows a detail of both antennas. The top antenna is a 9-element $824-960 \mathrm{MHz}$ directional $13 \mathrm{dBi}$ gain antenna whereas the bottom antenna is a 7-element $390-480 \mathrm{MHz}$ directional $10 \mathrm{dBi}$ antenna, both from the LPRS (Low Power Radio Solutions) company [10]. The antennas are mounted approximately $8 \mathrm{~m}$ above ground level, corresponding to $1390 \mathrm{~m}$ above sea level. The expeditions performed with the mobile vehicle always stay within the main beam width of these Yagi-Uda antennas. The gain antennas are only used for receiving, hence the power limits imposed by the different spectrum regulations in the world are not exceeded. However, as no spectrum regulation exists in the Antarctic, gain antennas could also be legally employed for transmitting, further extending the link's range. The receiver, based on the RN2483 chip which is specified to operate at temperatures down to $-40^{\circ} \mathrm{C}$, is mounted in a small cabinet near the antennas to limit coaxial cable losses, as is clearly visible in Fig. 2.

\section{Mobile transmitting setup}

The mobile vehicle used for the expeditions is a caterpillartrack equipped Toyota Hilux, as displayed in Fig. 3. Omnidirectional vertical monopole antennas for each band are installed on the roof of the vehicle. Similar hardware as in the base station is used as a transmitter. However, the transmitter now is controlled by a Raspberry PI board [11] in order to keep the power consumption as low as possible. Additionally, this board is specified to operate down to temperatures as low as $-40^{\circ} \mathrm{C}$. Measurements are performed during inspection of science setups up to a range of approximately $30 \mathrm{~km}$ from the base station.

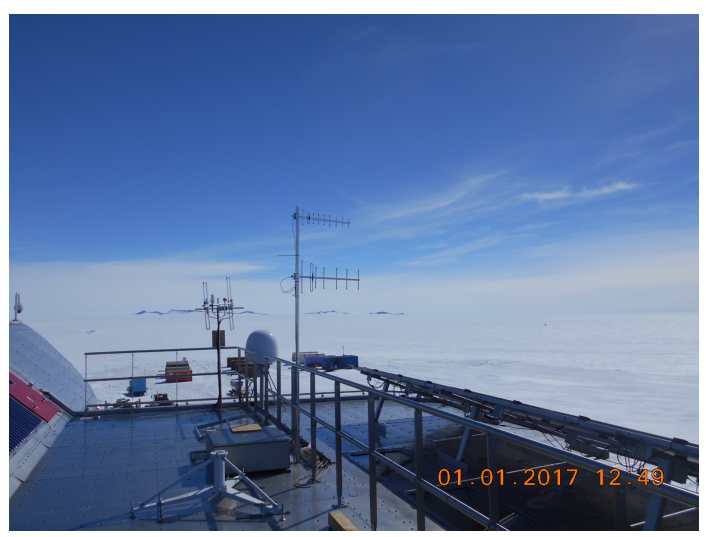

Fig. 1. Base station, equipped with Yagi-Uda receive antennas on a small mast.

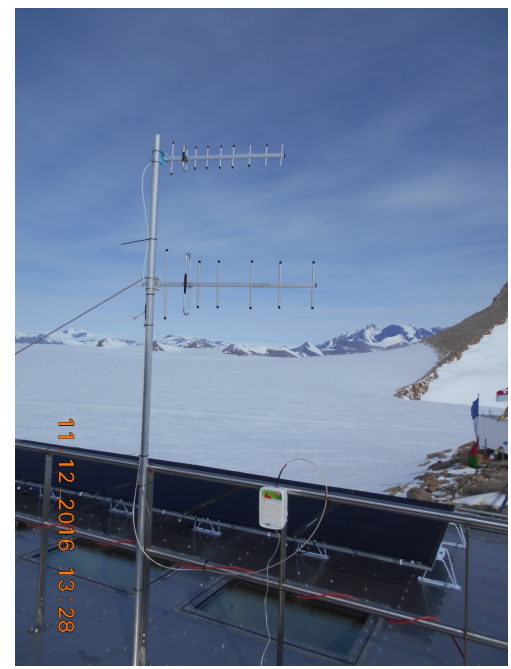

Fig. 2. Detail of the base station Yagi-Uda antennas and the LoRa receiver

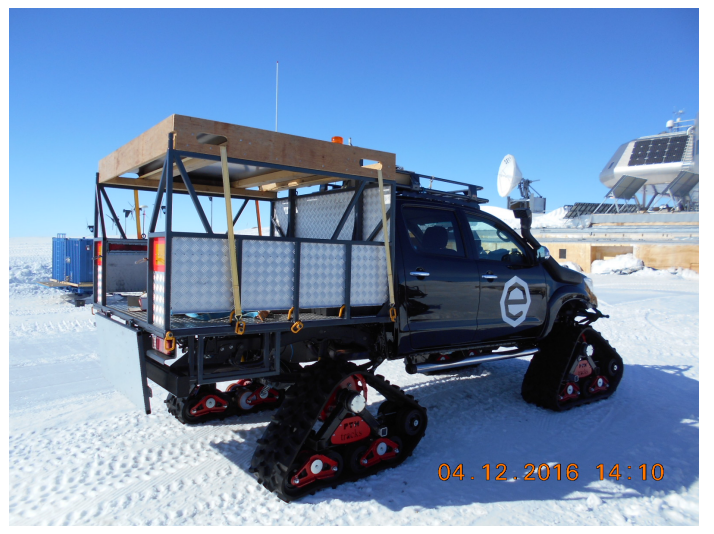

Fig. 3. Mobile vehicle, equipped with transmit antennas installed on the roof of the car. 


\section{MeAsurement RESUlts}

A number of measurements have been performed, during expeditions on different days. The LoRa system was further developed during the measurement campaign. Therefore initial measurements are only in the $868 \mathrm{MHz}$ band, further measurements are dual band, on 434 and $868 \mathrm{MHz}$. Some technical problems also limited the amount of useful data that was recorded. Due to the weather conditions, some measurements could not be repeated. Table I lists the available measurements.

TABLE I

ANTARCTIC LORA MEASUREMENTS

\begin{tabular}{|c|c|c|c|}
\hline Meas. & Date & Band (MHz) & Reference \\
\hline I & Dec. 17th, 2016 & 868 & - \\
II & Jan. 03th, 2017 & $434+868$ & - \\
III & Jan. 10th, 2017 & $434+868$ & {$[5]$} \\
IV & Jan. 20th, 2017 & $(434)+868$ & - \\
\hline
\end{tabular}

\section{A. Outbound and return path to base station at $868 \mathrm{MHz}$}

Measurement I was performed on $868 \mathrm{MHz}$ only. An expedition drove up to $35 \mathrm{~km}$ uphill from the base station, returning along almost the same trajectory. The GPS altitude recorded during the expedition is shown in Fig. 4 and is similar for the outbound and the return path, as a function of distance from the base station. The elevation angle relative to the base station varies between $-0.35^{\circ}$ and $+1.59^{\circ}$, well within the beam width of the receiving Yagi-Uda antenna. In azimuth, the receiving antenna is positioned in the direction of the trajectory, which is performed in a straight line, away from the base station.

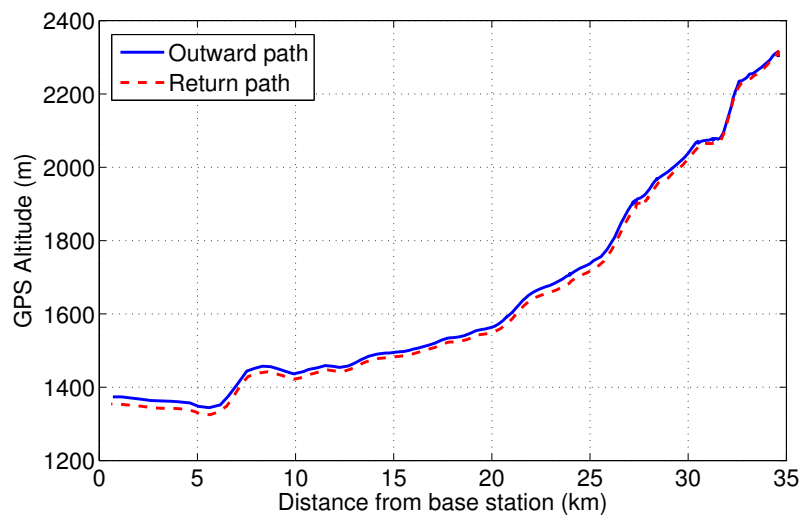

Fig. 4. Measurement I. GPS altitude recorded, for the outbound and return path along a similar trajectory.

The SNR recorded for a LoRa transmission along the trajectory is displayed in Fig. 5. At ranges up to $8 \mathrm{~km}$ the detector is saturated and packet loss is not present. Above this range, the detector is within its normal operating region. Due to the varying terrain elevation, the vehicle goes in and out of Line of Sight $(\mathrm{LoS})$ and large signal variations are recorded. These variations are partially attributed to ground reflections, interfering constructively and destructively with the direct signal. Because of the terrain profile, as visible in Fig. 4, the influence of reflections is higher than on flat ground. At larger ranges the average signal clearly decreases due to the path loss, and for distances above $25 \mathrm{~km}$ no packets are received anymore. Note that when no packets are received, the LoRa receiver keeps outputting the last recorded SNR value. Therefore those SNR values, corresponding to ranges between 25 and $35 \mathrm{~km}$ are not shown in the plot.

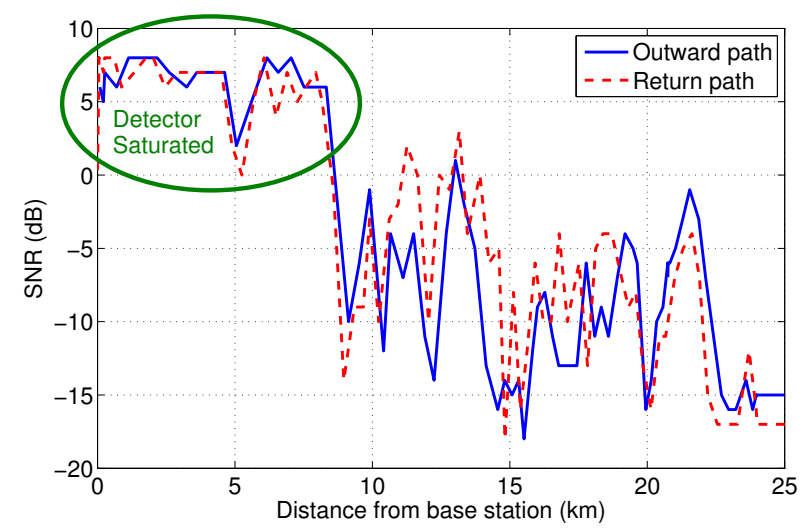

Fig. 5. Measurement I. SNR recorded at $868 \mathrm{MHz}$ for a first expedition, for the outbound and return path along a similar trajectory.

Signal fluctuations caused by the ground reflection are not correlated for the outbound and return path, because of minor differences in trajectory. As the wavelength $\lambda$ at $868 \mathrm{MHz}$ is around $35 \mathrm{~cm}$, maxima and minima are spatially as close as $\lambda / 4$ from each other. However, the path loss is highly similar for both curves. In order to fit a path loss characteristic to the measurements, only the data recorded with the detector within its normal operation region are selected. This corresponds to all signals received at ranges from 8 to $25 \mathrm{~km}$. The largest signal variations are first averaged by grouping the measurements per kilometer distance. The resulting curves are plotted in Fig. 6, where a similar trend for both curves becomes apparent. Now logarithmic path loss characteristics are derived for the SNR as a function of $\log _{10}(d)$, where $d$ is the distance in meter. For the outbound path, the SNR as a function of distance is given as

$$
\mathrm{SNR}=-26.6 \cdot \log _{10}(d)+103.5 \mathrm{~dB}
$$

The resulting outbound path loss PL is given as

$$
P L(d)=P L\left(d_{0}\right)+10 \cdot n \cdot \log _{10}\left(\frac{d}{d_{0}}\right),
$$

with the path loss exponent $n=2.66$ and $d_{0}=1 \mathrm{~m}$.

For the return path

$$
\mathrm{SNR}=-31.9 \cdot \log _{10}(d)+126.6 \mathrm{~dB}
$$

and hence $n=3.19$ in the resulting path loss equation. Outdoor path loss exponents in the inhabited world are known to be $2.7-3.5$ in urban environments and $3-5$ in suburban environments [12]. 


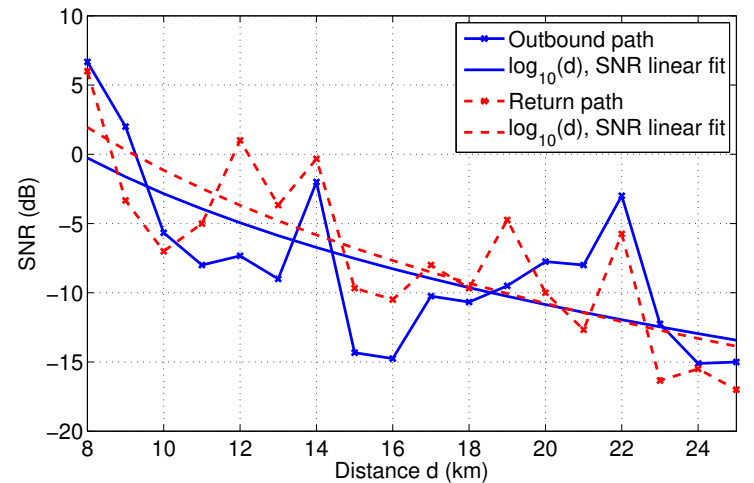

Fig. 6. Measurement I. SNR recorded at $868 \mathrm{MHz}$ for a first expedition, for the outbound and return path along a similar trajectory. The SNR values are grouped per $\mathrm{km}$ range and averaged. Path loss characteristic fitted to the measured curves.

\section{B. Comparison of 434 versus $868 \mathrm{MHz}$ signals}

Measurements II, III and IV were performed in two bands in order to determine the performance difference between $434 \mathrm{MHz}$ and $868 \mathrm{MHz}$ links. In measurement IV, the $434 \mathrm{MHz}$ link dropped out early in the measurement. Therefore, only measurement II and III can be analyzed for a comparison of both bands.

The scatter plot in Fig. 7 displays the received SNRs for both bands during measurements in which packets were transmitted from the mobile vehicle, alternating on 434 and $868 \mathrm{MHz}$, with an interval of 5 seconds. Two such measurements were performed along almost similar trajectories, on different days.

Note SNRs above $+2 \mathrm{~dB}$ are not included in the scatter plot, because these values are known to cause saturation of the detector. A linear fit, employing SNR values in $\mathrm{dB}$ for both measurements, provides slopes equal to 0.68 and 0.52 , for measurements II and III, respectively. The slopes appear fairly similar, however, the offset between both characteristics is as large as $13.2 \mathrm{~dB}$, which will be explained further. Note only the points corresponding to valid dual band SNR pairs, measured subsequently and within the linear detector range for both bands, are shown in the graph.

The statistics of the measurements, as presented in Fig. 8 reveal interesting information. The lower values recorded in measurement II for $434 \mathrm{MHz}$, and in particular the absence of the highest values normally recorded at close range to the base station, suggest a potential problem due to the antenna being too close to the vehicle frame. This results in extra losses for this band, possibly explaining the large difference in average signal level, combined with a similar slope.

The histograms as well as the analysis in [5] indicate the validity of measurement III. In this measurement, the average signal to noise ratio is $7 \mathrm{~dB}$ higher at $434 \mathrm{MHz}$, compared to $868 \mathrm{MHz}$, despite the fact that $3 \mathrm{~dB}$ more antenna gain is present at the base station receiver for $868 \mathrm{MHz}$. Clearly, the $434 \mathrm{MHz}$ band offers the most favorable propagation.

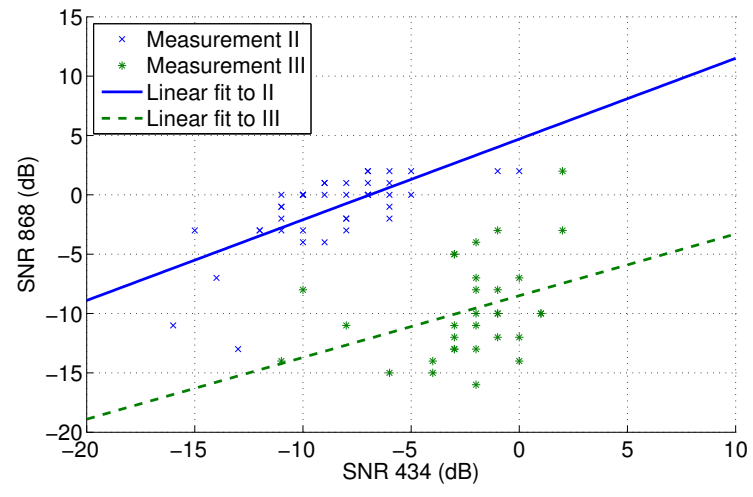

Fig. 7. Measurement II and III. SNR recorded at $434 \mathrm{MHz}$ and $868 \mathrm{MHz}$ during dual band measurements. Comparison for equal trajectories on different days.
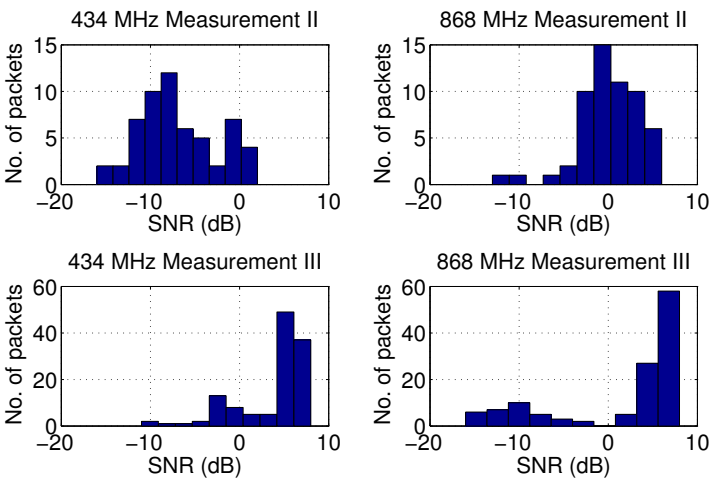

Fig. 8. Measurement II and III. Histogram of the SNR for measurements performed in different bands on different days.

\section{Packet loss and SNR at $28 \mathrm{~km}$ range for $868 \mathrm{MHz}$}

In measurement III, data were recorded for trajectories traveling up to $28 \mathrm{~km}$ from the base station and then moving several times between two science setups at this maximum range. The detector at the receiver properly detects the Signal to Noise Ratio (SNR) in the range of -20 to $+5 \mathrm{~dB}$. Above this range the detector is saturated, whereas below this range packets are not received anymore. Saturation of the detector occurs at close range, corresponding to a region with a radius up to $7 \mathrm{~km}$ from the base station where the communication is perfect and no packet loss is observed at all. A valid average SNR is available for all ranges and packet loss can hence be correctly analyzed as a function of SNR.

The SNR and packet loss at maximum range, while traveling between two science setups near each other, at a range of $28 \mathrm{~km}$ from the base station is displayed in Fig. 9. In total, 795 packets are received, whereas according to the sequence numbers 1838 packets were transmitted by the transmitter, resulting in an average packet loss of $56.75 \%$ over the complete measurement at maximum range. Measurement III provides the largest number of measurement points for packet loss calculations. The packet loss displayed in the graph is calcu- 
lated as a moving average over 30 valid received packets. The number of lost packets is determined by means of the missing sequence numbers at the receiving side. Clearly, packet loss fluctuates very significantly according to the position of the vehicle, local terrain elevation and possibly operations performed by the scientist at the setups, blocking the signal path. However, a limited throughput is always available and during the peaks of highest SNR, the packet loss is as low as $3 \%$, despite the $28 \mathrm{~km}$ range covered.

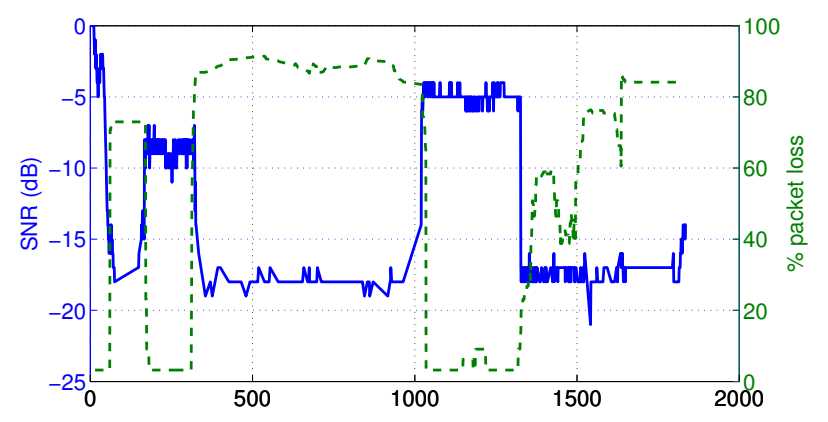

Fig. 9. Measurement III. Moving average of the packet loss at $28 \mathrm{~km}$ range for $868 \mathrm{MHz}$

A bar graph of the packet loss as a function of the average SNR expressed in $\mathrm{dB}$ per group of 30 received packets is shown in Fig. 10. Thanks to the spreading gain of the CSS modulation employed for LoRa communication, packet loss occurs only for received signal powers below the noise level. For higher SNRs, no packet loss is observed. A linear fit to the bar graphs allows to approximate the percentage of packet loss as $-5.4 \cdot \mathrm{SNR}-19$ for $-19 \mathrm{~dB}<\mathrm{SNR}<-3.52 \mathrm{~dB}$.

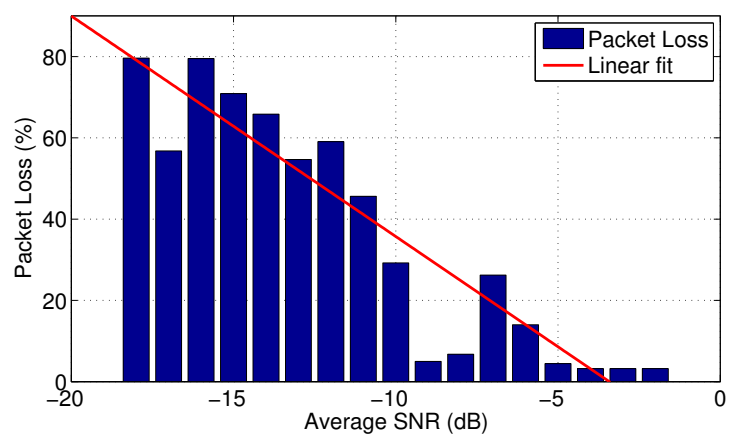

Fig. 10. Measurement III. Grouped packet loss as a function of SNR, for $868 \mathrm{MHz}$ at $28 \mathrm{~km}$ range.

The CSS modulation employed by the LoRa standard clearly causes a higher practical link budget. Frequency Shift Keying (FSK), as employed in Sigfox, and Bi-Phase Shift Keying (BPSK) in DASH7, require positive SNRs in $\mathrm{dB}$ to achieve similar packet loss figures at the receiver. Theoretically, an SNR of $0 \mathrm{~dB}$ results in a Bit Error Rate (BER) of 0.1482 for FSK and 0.0786 for BPSK, respectively. A BER in this order of magnitude would generally be uncorrectable by coding and result in an unacceptable packet loss rate.

\section{CONClusion}

The measurements performed in the Antarctic provide a clear indication of the practical range and reliability of LoRa wireless sensor links. Measurement results are shown to be reproducible, based on signal levels recorded during one expedition, comparing values recorded along the outbound and return path along the same trajectory.

The performance at 434 and $868 \mathrm{MHz}$ is compared. The $434 \mathrm{MHz}$ link generally provides a better signal to noise ratio, as expected based on wave propagation theory. Excluding saturation of the detector, the average signal to noise ratio is $7 \mathrm{~dB}$ higher at $434 \mathrm{MHz}$, compared to $868 \mathrm{MHz}$, despite the fact that $3 \mathrm{~dB}$ more antenna gain is present at the base station receiver for $868 \mathrm{MHz}$.

At $28 \mathrm{~km}$ range, a variable packet loss was measured. Depending on the position of the vehicle, packet loss values as low as $3 \%$ are possible. Packet loss was also characterized as a function of signal to noise ratio. The measurements indicate that LoRa communication over this range is perfectly possible.

The paper provided measurement and analysis of practical LoRa wireless sensor communication in the Antarctic. The results from this work will be used for the future development of wireless links to remote weather stations. Future research can also include topologies where the nodes not only transmit their own data, but additionally forward LoRa packets from more remote sensor nodes, in order to extend the range of the wireless sensor system.

\section{ACKNOWLEDGMENT}

This research was possible thanks to International Polar Foundation (www.polarfoundation.org / www.antarcticstation.org). Funding of the part of research infrastructure used in this paper was made available by BELSPO through the IAP Phase VII BESTCOM project.

\section{REFERENCES}

[1] Princess Elisabeth Antarctic Station http://www.antarcticstation.org, Accessed May 10, 2017.

[2] Lora Alliance, Lora alliance https://www.lora-alliance.org/.

[3] Sigfox.com, Sigfox https://www.sigfox.com/en.

[4] DASH7 Alliance, Dash7 http://www.dash7-alliance.org/.

[5] J. Gaelens, P. Van Torre, J. Verhaevert, and H. Rogier, "LoRa mobileto-base-station channel characterization in the Antarctic," Sensors, vol. 17, no. 8, 2017. [Online]. Available: http://www.mdpi.com/1424$8220 / 17 / 8 / 1903$

[6] J. B. Andersen, T. S. Rappaport, and S. Yoshida, "Propagation measurements and models for wireless communications channels," IEEE Communications Magazine, vol. 33, no. 1, pp. 42-49, Jan 1995.

[7] M. Lauridsen, B. Vejlgaard, I. Kovcs, H. Nguyen, and P. Mogensen, Interference Measurements in the European $868 \mathrm{MHz}$ ISM Band with Focus on LoRa and SigFox, 32017.

[8] J. Petjjrvi, K. Mikhaylov, M. Pettissalo, J. Janhunen, and J. Iinatti, "Performance of a low-power wide-area network based on LoRa technology: Doppler robustness, scalability, and coverage," International Journal of Distributed Sensor Networks, vol. 13, no. 3, 2017.

[9] Microchip, Microchip LoRa board DM164138 http://www.microchip.com/Developmenttools.

[10] LPRS, Low Power Radio Solutions Ltd http://www.lprs.co.uk.

[11] Raspberry Pi Foundation, Raspberry Pi https://www.raspberrypi.org/.

[12] T. S. Rappaport, Wireless Communications: Principles and Practice. Upper Saddle River, New Jersey: Prentice Hall PTR, 1996. 